\title{
Alexithymia, Physiological Reactivity and Cognitive Appraisals of Emotional Stimuli in Opiate Dependents: A Pilot Study.
}

\author{
Marco Torrado ${ }^{1^{*}}$, Hugo Silva ${ }^{2}$, Susana Eusébio ${ }^{3}$, Ana Fred ${ }^{4}$ and Silvia Ouakinin ${ }^{5}$ \\ ${ }^{1}$ Department of Psychiatry and Medical Psychology University Clinics, Portugal \\ ${ }^{2}$ Technical Institute, University of Lisbon, Portugal \\ ${ }^{3}$ University of Lisbon, Portugal \\ ${ }^{4}$ University of Lisbon, Portugal \\ ${ }^{5}$ Department of Psychiatry and Medical Psychology University, Portugal
}

*Corresponding author: Marco Torrado, Invited Professor, Faculty of Medicine, University of Lisbon, Medical Psychology, Av. Prof. Egas Moniz, Hospital Santa Maria 1649-028 Lisboa Lisboa, Portugal; E-mail: marcovtorrado@gmail.com

Received date: Oct 17, 2014, Accepted date: Dec 29, 2014, Published date: Jan 05, 2015

Copyright: ( 2014 Torrado M, et al. This is an open-access article distributed under the terms of the Creative Commons Attribution License, which permits unrestricted use, distribution, and reproduction in any medium, provided the original author and source are credited.

\begin{abstract}
Objective: The aim of this study was to investigate psychophysiological reactivity to daily-life stimuli in opiate dependents and associations with alexithymia. Methods: The sample $(n=72)$ was composed of a heroin addicts group treated with methadone and a healthy group, studied in several variables. Participants completed the Portuguese versions of the Toronto Alexithymia Scale-20 items, the Hospital Anxiety and Depression Scale and the Mini-Mental State Examination. They were also exposed to images from The International Affective Pictures System (IAPS) and physiological parameters and ratings of valence and arousal were collected. Results: Opiate dependents showed more alexithymia than controls and higher autonomic reactivity to stimuli. Higher levels of alexithymia seems to moderate an inverse association between heart rate and cognitive appraisals of unpleasant stimuli in this sample. Conclusion: A dysregulated brain-peripheral integration increased by the long term-use of drugs and damage in prefrontal regions are discussed as potential etiological factors supporting the presence of emotional deficits in these populations.
\end{abstract}

Keywords: Alexithymia; Psychophysiological parameters; Emotion; Valence; Arousal; Prefrontal cortex

\section{Introduction}

Studies pointed out through decades the presence of emotional regulation deficits in opiate dependents. From different theoretical perspectives some authors have suggested these individuals show selfregulation characteristics that inhibit healthy patterns of experiencing, identifying or expressing emotions, related to the long-term use of drugs [1-3]. Others have hypothesized that some of these dysfunctional emotional patterns are previous to drug abuse $[4,5]$. Although some studies have characterized these populations on constructs related to emotional regulation deficits, such as alexithymia or emotional awareness [6], few investigations have examined the emotion appraisals in clinical populations of substance users regarding daily-life stimuli which are motivationally relevant for people in general, and not related to drug use [7,8]. Additionally, no studies have focused on the associations between self-reported experiences of the emotional valence and arousal of stimuli and peripheral physiological reactivity to the same stimuli in these particular populations.

There are different models for studying emotions. More recently, some studies have focused on a dimensional perspective of the emotional functioning, contrasting with the discrete perspective, which contends that each emotion (e.g., anger, happiness) corresponds to a unique profile in experience, physiology and behavior [9]. According to the dimensional and motivational perspective, emotional responses are based on a few fundamental dimensions, such as valence and arousal. The valence dimension involves a continuum of states between happiness/pleasure to sadness/displeasure and the arousal element contrasts states of low bodily activation with others of high arousal/excitement. A third dimension termed as dominance was proposed [10], however later research did not recognized it as being as relevant as the other two dimensions, accounting poorly for the variance in evaluative judgments of symbolic stimuli $[11,12]$.

Research settings to elicit emotional states have used internationally well-validated assessment systems such as The International Affective Picture System (IAPS), developed by Lang and colleagues [13-15], based on a large number of images representing multiple situations, people, animals, nature, objects, that includes a significant set of emotional categories. Self-report scales (e.g. the Self-Assessment Manikin) are usually used to describe their subjective experiences of valence and arousal facing those collected stimuli and multiples studies conducted by Bradley and Lang [12,13] have shown linear relations between autonomic measures and these self-reports of emotional experience (skin conductance - arousal; and heart rate valence) in general population samples.

Nevertheless, few studies have focused on the experience of emotions in addicted samples, which are frequently assumed as dysfunctional on emotional regulation patterns across multiple domains (e.g. biological, psychological). So, this population may require additional assessments, especially concerning to the physiological aspects of the emotional responses.

Studies have suggested that heroin dependents differ from healthy controls in experiencing emotions, showing lower activations in response to highly pleasant arousing images and a greater response to 
neutral and unpleasant stimuli $[8-16,17]$. They suggest that drug abuse can produce long-term changes in the way drug users experience emotions facing natural stimuli. These changes in emotional appraisals seem to be long-standing, with no significant variance according to age, the chronicity of abuse or the length of the abstinence period [18]. Recent neuroimaging studies showed a lower cerebral activation in drug users when they faced emotional stimuli reported by normal population as pleasant or unpleasant, but a significant activation when videos related to drug use are shown [19]. It has been proposed that a sensitization of the motivational circuits toward stimuli associated with drugs could cause an inhibition of the emotional response to other natural reinforcers not related to drug use [8].

Research has also pointed that addicted people frequently show a cognitive-emotional style characterized by difficulties in identifying and describing feelings, which is related to dysfunctional mechanisms in the prefrontal cortex [20]. In a large review based on multiple studies, Larsen and colleagues [21] suggest that there are many distinct regions in the ventromedial prefrontal cortex, such as the anterior cingulate cortex and the orbitofrontal cortex, which seem to participate in emotion and to be associated with impairments in both the cognitive and emotional dimensions of alexithymia. The stimulation of some of these areas is also related to major autonomic responses. Damage in the prefrontal regions (e.g. orbitofrontal cortex) may generate dysregulation of the peripheral responses of emotion (e.g. lower or inflated expression of affect, blunted emotional experience) and provide a disturbed emotional processing compatible with the cognitive features of alexithymia.

However, the discussion about whether alexithymia is related to higher or lower levels of autonomic activity is unfinished due to the multiplicity of findings in research. Some studies emphasized associations between alexithymia and higher baseline levels of heart rate variability [22], heart rate [23] and skin conductance [24]. Others found either hypoarousal or hyperarousal when individuals were exposed to stressors or in recovery from those stimuli [25], suggesting possible different dimensions of alexithymia involved in different neural dysfunction, resulting in distinctive levels of sympathetic reactivity in healthy samples.

There is a relative lack of evidence concerning the autonomic correlates of emotional reactivity in opiate dependents, perhaps due to major difficulties in assessing these clinical populations. Research has emphasized a selective sympathetic reactivity to opiate-related stimuli, even in heroin-abstinent groups, which is not accompanied by a similar pattern when these individuals are exposed to neutral images or videos [26]. Methadone seems to provide in these individuals a modulation effect on autonomic function, increasing their low cardiac reactivity patterns immediately after taking it [27], but few investigations have focused on how individuals in long-term methadone maintenance experience emotions subjectively and physiologically. It is relevant to study the emotion recognition and experiencing patterns in drug dependents because of their significance in guaranteeing the course of rehabilitation. Dysfunctional patterns of experiencing and identifying emotions may induce maladaptive ways of dealing with daily events, compromising the recognition of relapse triggers and the abstinence itself [8].

In this study we assessed a sample of opiate dependents included in outpatient treatment centers and a control group, expecting to add more evidence on these issues. We hypothesized that opiate dependents report more alexithymia than controls, which would be related to decreased autonomic reactivity and to less accurate appraisals of valence and arousal of the emotional stimuli.

\section{Methodology}

\section{Participants}

The sample included 72 subjects, a heroin dependents group (DG) and a healthy group (HG). The first consisted of 46 heroin dependents collected in a treatment facility from the Service of Intervention on Addictive Behaviors and Dependencies (SICAD, Ministry of Health), in the city of Lisbon. Inclusion criteria were heroin-dependence medically stabilized in a methadone substitution program, with a minimum of four years of school and age between 18 and 65 years old. All participants were also followed in regular appointments with a therapist (psychiatrist or clinical psychologist) in individual psychotherapy, group therapy or both. We excluded participants that were taking psychotropic medication, with illicit opiates use in the last three months, presence of psychiatric co-morbidity such as mood disorders (major depressive or bipolar diseases), psychotic disorders as well as subjects with markedly cognitive deficits. Those with severe medical conditions such as HIV infection, non-treated hepatitis or oncologic diseases were also excluded.

The healthy subjects group (HG) consisted of 26 individuals, collected in a public facility for blood-donation, in Lisbon city. All participants were healthy, with a minimum of four years of school and age between 18 and 65 years old, with no complaints or history of psychiatric conditions or other diseases. Participants who were doing their first blood-donation were excluded.

This study was approved by the Ethical Boards of the treatment' and blood-donors facilities. All participants gave their written informed consent.

\section{Instruments}

Participants were initially assessed using a semi-structured interview, including socio-demographic, medical and drug-use variables, the Portuguese versions of the Toronto Alexithymia Scale 20 items [28], the Mini-Mental State Examination [29] and finally the Hospital Anxiety and Depression Scale [30] as a screening for cognitive deficits and states of anxiety and depression, respectively. In a second session a specific experimental setting was carried out, in which participants were exposed to a selection of twelve images from The International Affective Pictures System, IAPS [15] and physiological parameters (heart rate, skin conductance and respiratory frequency) were collected using a bioPLUX research device (PLUX ๑). Participants were also asked to rate valence and arousal of the IAPS pictures presented through the Self-Assessment Manikin [31].

The Toronto Alexithymia Scale (TAS-20) is the most used measure of alexithymia in several countries and languages. It is a self-report scale with twenty items rated from 1 (strongly disagree) to 5 (strongly agree). Examples of the items are the following: "I am often confused about what emotion I am feeling"; "It is difficult for me to reveal my innermost feelings even to close friends". The sum of all 20 items, taking reversed items into account, was used to produce a total score. Confirmatory factor-analysis of the Portuguese version supports the three-factor model of the original scale ('difficulty identifying feelings', DIF; 'difficulty describing feelings', DDF and 'externally oriented thinking', EOT). Values for internal consistency (Cronbach's alpha, a 
$=.79)$ and test-retest stability over time $(r=.90$ for three weeks between two assessment moments, $\mathrm{p}<.001)$ confirm the very good reliability levels of this version [27].

The International Affective Picture System (IAPS) is a large set of emotionally-evocative pictures / photographs that includes contents across a wide range of semantic categories. These images are classified according to three relevant dimensions (valence, arousal and dominance) and each image has available normative values for those dimensions [15]. Based on the valence and arousal norms of IAPS, the selected images were arranged to form four conditions that differ in their affective value (highly arousing/pleasant, highly arousing/ unpleasant, less arousing/pleasant and less arousing/unpleasant pictures). Each condition had three images. Due to high variability between genders for highly arousing pictures, we decide to choose a different picture for men and women among the three. The other conditions were the same to both genders.

Each of the 12 IAPS pictures was shown for $6 \mathrm{~s}$, separated by a 1-s period of visual fixation (a cross-hair appeared on a black screen).

The bioPLUX research device is composed of a set of sensors (the respPLUX, bvpPLUX and edaPLUX) for measurement of respiratory frequency (RESP), blood volume pulse photoplethysmography (BVP) and electrodermal activity (EDA), with 12-bit resolution and $1000 \mathrm{~Hz}$ sampling rate per channel. It used a light-dependent resistor (LDR) sensor to assure synchronization between the content presented on the screen and the physiological data being acquired.

The Self-Assessment Manikin (SAM) is a visual-analogical scale and it was specifically designed to measure the subjective responses to IAPS images. It is used to collect self-reported information about emotional experience in the three relevant dimensions described earlier, in Lang's model. In this study we focused particularly on two dimensions (valence and arousal). The SAM provides scores within a $0-9$ range $(0=$ very unpleasant and 9 very pleasant, for valence; $0=$ very calm and $9=$ very arousing, for arousal).

\section{Procedures}

The assessment of DG participants was carried out after their methadone taking and before the appointment with their therapist. HG subjects were invited to participate in this investigation after the medical screening and before the blood donation. In the second evaluation session, before exposing the subjects to the full set of IAPS pictures they were connected to the bioPLUX research device. They were assessed in a resting period about two minutes and during the IAPS exposure.

Subjects were given this instruction: "Please look at the screen and allow yourself to feel whatever each image evokes in you." Immediately after the set of pictures had just been shown, they were replayed in the same order and ratings of valence and arousal for each picture were obtained through the SAM, based on how subjects recalled experiencing the content of pictures during the first viewing minutes before.

\section{Data Reduction and Analyses}

The psychophysiological parameters used were chosen based on the literature [31,32] according to their relevance and similar experimental setups. We also analyzed additional features that we thought could also be discriminative of each population.

For feature selection, the raw data were processed using the Python programming language, and the SciPy scientific computing library. The raw data was first filtered to eliminate artifacts such as powerline interference; the BVP signal is bandpass filtered with a [1-8] $\mathrm{Hz}$ passing band, using a 4 th order filter, the raw EDA signal is lowpass filtered with a $0.25 \mathrm{~Hz}$ cutoff frequency, using a 2nd order filter, and the raw RESP data is bandpass filtered with a [0.10-0.35] $\mathrm{Hz}$ passband, also using a 2nd order filter. For all signals, a Butterworth filter design was used since it is known to provide the best impulse response in the cutoff frequency boundaries, and a zero-phase forward and reverse digital filtering process was adopted [34,35].

Statistical analyses were generally conducted with the Statistical Package for the Social Sciences (SPSS), version 19 for Macintosh. All statistical tests were two-tailed. A $5 \%$ significance level was usually considered, although exceptionally a $10 \%$ level was indicated, as a reference for future studies of a potential clinical importance to be analyzed. Comparisons between independent samples and associations between dependent variables were performed through Mann-Whitney Non-Parametric Tests and Spearman Correlations or associated significance tests, respectively, since assumptions of normality of variables were not met. Moderation effects were also tested on the association between specific independent and dependent variables using multiple regression analysis where a moderator effect was added as an interaction term between a pair of independent variables [36].

\section{Results}

DG participants were integrated for overall 68 months in methadone program $(M=50,74 \mathrm{mg} ; \mathrm{SD}=28,49)$. Groups were homogenous on socio-demographic variables considered (i.e. marital status, employment situation) but different in terms of age and educational level, which constitute limitations of the study. Although groups also have different gender distribution, when considering each group separately and testing for differences between genders regarding all studied variables no differences were found except for the appreciation of low arousing and negative stimuli. States of anxiety and depression were below the cut-off. Both groups did not show significant differences in MMSE scores, which were within normal ranges, suggesting the absence of severe cognitive deficits (Table 1).

\begin{tabular}{|l|l|l|l|l|}
\hline Mean ranks & DG $(n=46)$ & HG $(n=26)$ & Mann-Whitney U & $p$ \\
\hline Age & 40,68 & 27,90 & 374,500 & $.012^{*}$ \\
\hline MMSE & 37,47 & 30,92 & 453,000 & .175 \\
\hline HADS-A & 36,60 & 32,35 & 490,000 & .391 \\
\hline HADS-D & 35,88 & 33,54 & 521,000 & .636 \\
\hline
\end{tabular}




\begin{tabular}{|c|c|c|c|c|}
\hline \multicolumn{3}{|l|}{$N$} & \multirow[t]{2}{*}{$x^{2}$} & \multirow[t]{2}{*}{$\mathrm{p}$} \\
\hline Gender & & & & \\
\hline Male & 30 & 7 & \multirow{2}{*}{352,500} & \multirow{2}{*}{$.001^{* *}$} \\
\hline Female & 15 & 19 & & \\
\hline \multicolumn{5}{|c|}{ Education level } \\
\hline Basic & 29 & 3 & \multirow{3}{*}{26,314} & \multirow{3}{*}{$.000^{* *}$} \\
\hline Secondary & 12 & 8 & & \\
\hline Higher & 4 & 15 & & \\
\hline \multicolumn{5}{|c|}{ Working status } \\
\hline employed & 34 & 20 & \multirow{2}{*}{6,588} & \multirow{2}{*}{.086} \\
\hline unemployed & 11 & 6 & & \\
\hline
\end{tabular}

Table 1: Description of the groups. ${ }^{\star} \mathrm{p}<.05 \quad{ }^{* *} \mathrm{p}<.01$

Results showed that heroin dependent individuals reported higher levels of alexithymia, DIF, DDF and EOT, even though not reaching a statistical significant difference in relation to controls. HG participants' alexithymia level was similar to the mean score found in studies with TAS-20 in Portuguese samples (HG, $n=26, M=47,65$; $\mathrm{SD}=13,99$; Portuguese sample from validation studies, $\mathrm{n}=298$, $\mathrm{M}=47,17 ; \mathrm{SD}=10,81$ ), whereas the alexithymia reported level by DG participants was higher (DG, $\mathrm{n}=45, \mathrm{M}=51,91 ; \mathrm{SD}=13,24)$. Alexithymia scores did not relate to age or methadone dose variables (rho=-.190, $\mathrm{p}=.113$; rho=.243, $\mathrm{p}=.112$ ).

DGs showed heart rate levels significantly elevated when facing all kinds of stimuli except the low arousing and negatives. The same was observed on their heart rate variation between baseline and the exposition to pleasurable arousing stimuli. Skin conductance responses for high arousing stimuli were also higher than controls, although a significant reduced skin conductance tonic level was observed at the baseline. Finally, the appraisal of valence for high arousing stimuli was also elevated, although with a p-value at the level of $10 \%$ (Table 2).

\begin{tabular}{|l|l|l|l|l|}
\hline \multicolumn{4}{|l|}{ Mean ranks for DG and HG and Mann-Whitney Test } \\
\hline $\begin{array}{l}\text { TAS-20, } \\
\text { bioPLUX and SAM }\end{array}$ & DG (n=46) & HG $(n=26)$ & $\begin{array}{l}\text { Mann- } \\
\text { Whitney U }\end{array}$ & $p$ \\
\hline TAS-20 Total & 38,57 & 31,56 & 469,50 & .168 \\
\hline DIF & 38,88 & 31,02 & 455,50 & .121 \\
\hline DDF & 37,77 & 32,94 & 505,50 & .342 \\
\hline EOT & 37,33 & 33,69 & 525,00 & .472 \\
\hline SCL Baseline & 32,31 & 42,38 & $419,00^{*}$ & .048 \\
\hline HR Baseline & 37,87 & 32,77 & 501,00 & .316 \\
\hline IRR Baseline & 37,02 & 34,23 & 539,00 & .583 \\
\hline SRR A+ V+ & 39,62 & 29,73 & $422,00 \dagger$ & .051 \\
\hline SRR A+ V- & 39,40 & 30,12 & $432,00 \dagger$ & .068 \\
\hline
\end{tabular}

\begin{tabular}{|l|l|l|l|l|}
\hline SRR A- V+ & 33,42 & 40,12 & 478,00 & .202 \\
\hline SRR A- V- & 33,04 & 41,12 & 452,00 & .112 \\
\hline HR A+ V+ & 40,33 & 28,50 & $390,00^{*}$ & .020 \\
\hline HR A+ V- & 39,71 & 29,58 & $418,00^{*}$ & .046 \\
\hline HR A- V+ & 40,18 & 28,77 & $397,00^{*}$ & .025 \\
\hline HR A- V- & 39,40 & 30,12 & $432,00 \dagger$ & .068 \\
\hline PHRI A+ V+ & 40,27 & 28,62 & $393,00^{*}$ & .022 \\
\hline PHRI A+ V - & 34,98 & 37,77 & 539,00 & .583 \\
\hline PHRI A- V+ & 41,09 & 27,19 & $356,00^{* *}$ & .006 \\
\hline PHRI A- V- & 38,29 & 32,04 & 482,00 & .219 \\
\hline SAM Valence A+ V+ & 39,16 & 30,54 & $443,00 \dagger$ & .087 \\
\hline SAM Valence A+ V- & 39,34 & 30,21 & $434,50 \dagger$ & .054 \\
\hline
\end{tabular}

Table 2: Differences between DG and HG participants on TAS-20, BioPLUX physiological parameters and SAM; TAS-20 Total - TAS-20 total score; DIF - difficulties identifying feelings; DDF - difficulties describing feelings; EOT - externally-oriented thinking; SCL Baseline tonic level of skin conductance measured at the resting period (previous to the set of images) by bioPLUX; HR Baseline - heart rate frequency measured at the resting period (previous to the set of images) by bioPLUX; IRR Baseline - respiratory frequency measured at the resting period (previous to the set of images) by bioPLUX; SRR $\mathrm{A}+\mathrm{V}+$ - skin conductance response rate (events/min.) for high arousing positive stimuli, measured by bioPLUX; SRR A+ V- - skin conductance response rate (events/min.) for high arousing negative stimuli, measured by bioPLUX; SRR A- V+ - skin conductance response rate (events/min.) for low arousing positive stimuli, measured by bioPLUX; SRR A- V- - skin conductance response rate (events/min.) for low arousing negative stimuli, measured by bioPLUX, HR A $+\mathrm{V}+-$ heart rate for high arousing positive stimuli measured by bioPLUX; HR A+ V- - heart rate for high arousing negative stimuli measured by bioPLUX; HR A- V+ - heart rate for low 
arousing positive stimuli measured by bioPLUX; HR A- V- - heart rate for low arousing negative stimuli measured by bioPLUX; PHRI A+ V+ - relative heart rate variation between baseline and stimuli (\%) for high arousing positive stimuli, measured by bioPLUX; PHRI A+ V- relative heart rate variation between baseline and stimuli (\%) for high arousing negative stimuli, measured by bioPLUX; PHRI A- V+ relative heart rate variation between baseline and stimuli (\%) for low arousing positive stimuli, measured by bioPLUX; PHRI A- V- relative heart rate variation between baseline and stimuli (\%) for low arousing negative stimuli, measured by bioPLUX; SAM Valence $\mathrm{A}+\mathrm{V}$ + - the appraisal of valence through Self-Assessment Manikin for high arousing positive stimuli; SAM Valence A+ V- - the appraisal of valence through Self-Assessment Manikin for high arousing negative stimuli; ${ }^{\star} \mathrm{p}<.05 \dagger \mathrm{p}<.10$

Table 3 highlights significant associations between alexithymia, physiological reactivity to stimuli and cognitive appraisals measured by SAM. In heroin dependents, results show correlations between emotional self-reported deficits and sympathetic-related parameters. Alexithymia was related to a lower respiratory rate at the baseline and EOT was negatively associated with electrodermal activity when subjects faced disturbing stimuli.

In controls, alexithymia correlated negatively with heart rate variation from the baseline when participants were viewing adverse stimuli (high arousing with negative valence). The DIF was negatively associated with a sympathetic parameter of skin conductance response.

Concerning the cognitive appraisals in opiate dependents, results showed alexithymia and DIF both related positively with appraisals of valence of negative arousing stimuli; and were negatively related with appraisals of valence of non-exciting positive stimuli. DDF correlated negatively with the valence evaluation of pleasurable stimuli. In controls, alexithymia and the TAS-20 dimensions correlated negatively with the ratings of valence of the positive stimuli, and also with the appraisals of low arousing images.

\begin{tabular}{|c|c|c|c|c|c|c|c|c|c|c|c|}
\hline \multicolumn{12}{|c|}{ BioPLUX and SAM } \\
\hline & \multicolumn{5}{|l|}{ DG } & \multicolumn{6}{|l|}{ HG } \\
\hline & \multicolumn{2}{|l|}{ BioPLUX } & \multicolumn{3}{|l|}{ SAM } & \multicolumn{3}{|c|}{ BioPLUX } & \multicolumn{3}{|l|}{ SAM } \\
\hline $\begin{array}{l}\text { TAS-20 } \\
\text { Scores }\end{array}$ & IRR Baseline & $\begin{array}{l}S R R \\
A+V-\end{array}$ & $\begin{array}{l}\text { SAM Val } \\
\mathrm{A}^{+} \mathrm{V}+\end{array}$ & $\begin{array}{l}\text { SAM Val } \\
\text { A- V+ }\end{array}$ & $\begin{array}{l}\text { SAM Val A+ } \\
\text { V- }\end{array}$ & $\begin{array}{l}S R R \\
A+V+\end{array}$ & $\begin{array}{l}\text { PHRI } \\
A+V-\end{array}$ & $\begin{array}{l}\text { SAM Val } \\
A+V+\end{array}$ & $\begin{array}{l}\text { SAM Val } \\
\text { A- V+ }\end{array}$ & $\begin{array}{l}\text { SAM Ar } \\
\text { A- }-{ }^{+}\end{array}$ & $\begin{array}{l}\text { SAM Ar } \\
\text { A- V- }\end{array}$ \\
\hline Total & $-.330^{*}$ & & & $-.350^{*}$ & $.335^{*}$ & & $-.438^{*}$ & & $-.440^{*}$ & $-.447^{*}$ & $-.403^{*}$ \\
\hline DIF & & & & $-.343^{*}$ & $.394^{\star *}$ & $-.418^{*}$ & & & & & $-.569^{\star *}$ \\
\hline DDF & & & $-.356^{*}$ & & & & $-.459^{*}$ & -.432 & $-.511^{* *}$ & & \\
\hline EOT & & $-.333^{*}$ & & & & & & & $-.414^{*}$ & & \\
\hline
\end{tabular}

Table 3: Significant correlations (Spearman) for DG and HG between TAS-20, bioPLUX parameters and SAM scores; Total - TAS-20 total score; DIF - difficulties identifying feelings; DDF - difficulties describing feelings; EOT - externally-oriented thinking; IRR Baseline - respiratory frequency measured at the resting period (previous to the set of images) by bioPLUX; SRR A+ V+ - skin conductance response rate (events/min.) for high arousing positive stimuli, measured by bioPLUX; SRR A+ V- - skin conductance response rate (events/min.) for high arousing negative stimuli, measured by bioPLUX; PHRI A+ V- - relative heart rate variation between baseline and stimuli (\%) for high arousing negative stimuli, measured by bioPLUX; SAM Valence A+ V+ - the appraisal of valence through Self-Assessment Manikin for high arousing positive stimuli; SAM Valence A+ V- - the appraisal of valence through Self-Assessment Manikin for high arousing negative stimuli; SAM Valence A- V+ - the appraisal of valence through Self-Assessment Manikin for low arousing positive stimuli; SAM Ar A- V+ - the appraisal of arousal through SelfAssessment Manikin for low arousing positive stimuli; SAM Ar A- V- - the appraisal of arousal through Self-Assessment Manikin for low arousing negative stimuli; $\mathrm{p}<.05 \quad{ }^{* *} \mathrm{p}<.01$

Whereas in controls cognitive self-reported appraisals of valence correlated with cardiovascular parameters - heart rate and heart rate variation - for some kinds of stimuli (low arousing negative stimuli (rho=-.420, p=.033; and rho=-.705, $\mathrm{p}=.000$; respectively), results also suggested that self-reported appraisals of valence and arousal are dissociated to the physiological parameters of reactivity to emotional stimuli in the drug dependents sample.

We tested if this lack of association in opiate dependents would be partially explained by a moderation effect of alexithymia. Specifically in the case of the association between appraisals of the valence of adverse stimuli and the respective heart rate measure, it was observed a relation almost null, close to zero, when alexithymia in opiate dependents is at a lower or at an intermediate level. However, the association between cognitive and cardiac reactivity is negative and very strong when opiate dependents show high levels of alexithymia (Figure 1). 


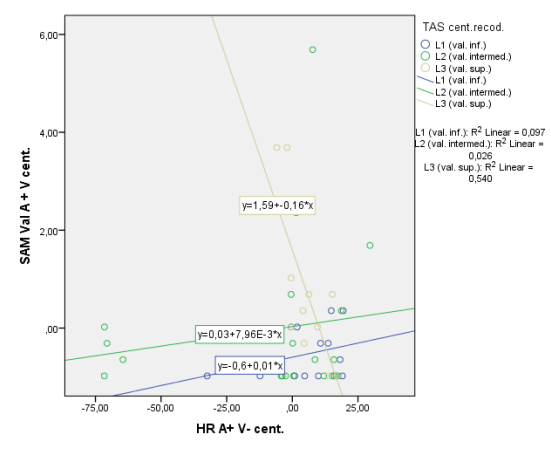

Figure 1: Moderation effects of alexithymia on the association between heart rate and valence appraisals for adverse stimuli.

\section{Discussion}

The aim of the present study was to provide new data for characterizing dimensions of emotional regulation at a psychological and psychophysiological level in opiate dependents, compared to healthy controls. Although caution is needed to conclude on the results, due to difficulties in matching cases and controls concerning age and education level, findings showed that a sample of drug users in abstinence for heroin appear to be more alexithymic than controls, and more impaired in their capacities for labeling and expressing feelings. They also show different patterns of reactivity to emotional stimuli, which seem to be associated with major difficulties in identifying and expressing emotions.

The clinical sample showed higher cardiovascular reactivity levels for the categories of stimuli considered, and higher levels of electrodermal responses and appraisals of valence for high arousing stimuli.

Regarding the amplified cardiovascular activity in opiate dependents, these results are relatively consistent with previous findings suggesting that people more alexithymic assessed in general population samples show higher heart rates than controls [37] which may be interpreted as supportive of a global elevated peripheral reactivity (heart rate is under both sympathetic and parasympathetic influences). This pattern can be related to several factors. Although abstinent from opiates, some participants reported occasional use of other drugs, which may increase cardiovascular activity. It is relevant to consider that heart rate levels shown may also be explained by a methadone effect. Participants were invited to join to this study after their methadone taking, which might have modulated their cardiac reactivity to emotional contents of the images and perhaps give some support to the increased variation between baseline and the presentation of the pleasurable stimuli compared to controls. Chang and collaborators [27] highlighted the protective effect of methadone in opiate dependents recently relapsed, increasing their heart rate levels and cardiac variability after taking it.

From other perspective, this pattern might have been established throughout the developmental life-course in these individuals, in which growing difficulties of emotion regulation could have drew a particular cognitive-affective style of processing emotional information accompanied by an autonomic dysregulation that, in some way, provided a more favorable context for contacting with drugs. These results can be read as consistent with a self-medication hypothesis for understanding the use of drugs [39, 40, 41]. In previous research [4] a developmental perspective of emotional dysregulation in these patients was suggested when we found significant higher levels of alexithymia in a cognitive non-impaired sample of opiate dependents $(n=99)$, compared to matched controls, and those emotion regulation deficits were strongly related with autobiographical reports of early parental rejection. Nevertheless, even if these individuals used opiates for decreasing an hypothetical previous amplified cardiac reactivity, results suggest that they still under-regulate their own emotional states, perhaps due the lack of cognitive strategies of emotional regulation, consistent with an alexithymic pattern.

The electrodermal responses shown by DG participants are higher for the arousing stimuli, despite their valence, supporting previous considerations about the sympathetic reactivity in these individuals. However, the skin conductance responses for the adverse images, higher than controls, seem to be inversely related to a specific characteristic of alexithymia, the externally-oriented thinking. This finding suggest that opiate dependents with this particular cognitive style may be less reactive to threatening contexts or stimuli, which is consistent with characteristics described in these populations (e.g. lack of self-care and self-protective behaviors) that are frequently related to relapse. This pattern also suggests a disturbed regulation of the prefrontal areas. An appropriate functioning of these cortical regions is vital to the detection of threats, maintenance of the integrity of the system, and therefore is crucial to health [38].

Results on SAM also suggest that these patients evaluate the arousing stimuli as more pleasurable (higher ratings of valence) despite their value of pleasantness (i.e. compared to ratings of people from general population), which may support difficulties to recognize threatening stimuli, to discriminate them from others and to behave properly. Additionally, self-reported valence appears to be related to alexithymia suggesting that this cognitive-affective style of emotional processing is associated with a less accurate evaluation of the pleasantness dimension for characterizing the stimuli (positive and negative blunting of the images, similar to an anhedonic functioning), which contrasts in some way with previous findings [16]. In healthy controls alexithymia also appears to be related to less accurate judgments of the pleasurable characteristics of positive stimuli, in a stronger way than in opiate dependents. In general population sample, higher alexithymic subjects seem additionally to identify non-arousing stimuli as more peaceful, supporting that alexithymic people perhaps show deeper difficulties in recognizing the arousal dimension of the events.

Findings also support dissociation between cognitive and physiological responses to emotional stimuli in DG participants on the studied parameters. Whereas controls show some significant correlations between subjective appraisals and autonomic parameters of reactivity (for low arousing negative stimuli) no significant correlations were met between self-reported emotional content of the stimuli and psychophysiological parameters. However, the negative correlation between cognitive and cardiac reactivity specifically moderated by high levels of alexithymia in opiate dependents, highlights the possible role of this affective style of functioning in a cognitive-autonomic dissociation (or disturbed association) which may contribute to the lack of self-regulation these individuals show. The observed pattern may point to a poor brain-peripheral (topdown) integration which is consistent with a possible damage of the circuits in prefrontal cerebral areas, several times reported in literature 
concerning to the drug addicted samples [42]. These impairments are described as strongly related to the long-term use and continuous exposure to opiates and other drugs (e.g. psychostimulants), resulting frequently in a decreased synaptic plasticity and drug-induced neuroadaptations [20]. Lesions in orbitofrontal and medial prefrontal regions have been associated with deficits in emotional appraisals, executive functions and particularly in decision-making in drug dependents, providing less inhibitory processes and related difficulties to evaluate daily-life situations and stimuli - particularly in stressful, aversive or drug-related contexts [19], which may play an important role in relapse. Physiological deficits in the same cerebral areas have been reported as associated to a disturbed emotional expression and autonomic dysregulation typical from alexithymia, compatible with both intense (or absence of) expression of affect or loss of inhibitions and a poor capacity to elaborate about the inner emotional states [21].

Thus, the current data seems to propose that the autonomic hyperactivity showed by DG participants when they viewed emotional images is decoupled from their cognitive appraisals. The evaluation of valence seems to be biased by the arousing level of the stimuli despite their positive or negative value and related to the presence of alexithymia. Perhaps this disinhibition of sympathoexcitatory circuits is linked with a prefrontal hypoactivation and limbic hyperactivation, carried out by a long period of exposure to drugs as some authors have suggested [42].

At the baseline, groups differed in the skin conductance tonic levels, which were higher for controls, and DG showed a respiratory rate negatively related to self-reported alexithymia. These results suggest that higher alexithymic opiate addicts show less sympathetic activity in resting, nevertheless further research is needed to support this assumption.

This study provided new information on emotional regulation deficits in heroin dependents, suggesting that these patients, even abstinent for illicit opiates, reported more difficulties to access their inner emotional states and to describe them than healthy people, although this research did not found a statistical difference between the studied groups. In terms of their autonomic reactivity, they seem to be less reactive in resting but over-reactive when visualize emotional stimuli, which may support difficulties to inhibit limbic information at a cortical level and regulate behavior properly. When these patients are more alexithymic those difficulties may be increased and related to blunted appraisals of adverse stimuli and to reduced competencies for evaluating pleasure in positive stimuli, in an anhedonic-like way. These patterns of functioning are probably associated in some way with the difficulties showed by these patients in discriminate pleasure in natural reinforcers from other types of stimuli, but also with the lack of self-care and self-protectiveness against threatening events that characterize some of these individuals. These aspects may be extremely relevant for planning adequate therapeutic interventions with these populations, namely with a focus on labeling emotions and differentiating the arousing from the valence dimensions of the events. Acknowledging that some experiences can be very exciting immediately, although very negative in terms of their potential consequences, can be extremely relevant for guaranteeing the continuity of a well-succeeded rehabilitation process with these patients.

Nevertheless, as mentioned, several limitations in this study have to be acknowledged. First, groups were different in relevant variables such as age and education level, which may have influenced the results. Second, heroin use is frequently combined with other drugs.
Autonomic parameters such as heart rate measures may have been influenced by other consumptions (e.g. alcohol, cigarettes), which were not accurately assessed in our subjects and, in some extent, differences between groups might be attributable to poly-substance use. Third, whether patients had been abstinent for other drugs recently was based on self-report. It would be safer to perform the urine drug screening to verify it objectively. Fourth, only anxiety and mood disorders were screened. A more structured interview to detect psychiatric symptoms would be useful for screening possible confounding comorbidities. Fifth, it cannot be completely excluded any methadone effects in the observed emotional reactivity of DG participants. Even so, besides the fact that individuals studied did not take high dosages of the agonist, previous research [43] pointed that methadone modulation is higher in peak plasma concentration (3 hours after taking it) that perhaps would not be the case of our sample in which subjects were assessed immediately after the methadone taking. Authors highlighted the relevance of taking into account an increased tolerance in individuals with a chronic use of opiates [43]. Finally, this study is based on a cross-sectional design and a small sample size, which limits further interpretations of the results. Larger samples and a longitudinal study are needed to support these findings.

Despite the abovementioned limitations these results may contribute to support the use of emotion regulation strategies in a psychotherapeutic approach. Some investigations [44] have underlined that emotion regulation training can be more effective than cognitive therapy for improving distress tolerance and adjustment, as well as in the control of drug abuse or misuse, providing a better adherence to pharmacotherapy.

\section{References}

1. Schuckit MA (2006) Comorbidity between substance use disorders and psychiatric conditions. Addiction 101 Suppl 1: 76-88.

2. Volkow N (2004) Drug dependence and addiction, III: Expectation and brain function in drug abuse. Am J Psychiatry 161: 621.

3. Kornreich C, Foisy M-F, Philippot P, Dan B, Tecco J et al. (2003) Impaired emotional facial expression recognition in alcoholics, opiate dependence subjects, methadone maintained subjects and mixed alcoholopiate antecedents subjects compared with normal controls. Psychiatry Research 119: 251-260.

4. Torrado M, Ouakinin S, Bacelar-Nicolau L (2013) Alexithymia, emotional awareness and perceived dysfunctional parental behaviors in heroin dependents. The International Journal of Mental Health and Addiction, 11:706-718.

5. Taylor J, Bagby RM., Parker J (1997) Disorders of Affect Regulation: Alexithymia in medical and psychiatric illness. Cambridge, Cambridge University Press.

6. Carton S, Bayard S, Paget V, Jouanne C, Varescon I, et al. (2010) Emotional awareness in substance-dependent patients. J Clin Psychol 66: 599-610.

7. Roedema TM, Simons RF (1999) Emotion-processing deficit in alexithymia. Psychophysiology 36: 379-387.

8. Aguilar de Arcos F, Verdejo-Garca A, Peralta-RamÃrez MI, SanchezBarrera M, Parez-Garca M (2005) Experience of emotions in substance abusers exposed to images containing neutral, positive, and negative affective stimuli. Drug and Alcohol Dependence 78:159-167.

9. Ekman P (1999) Basic emotions. In: Dalgleish T, Power MJ. eds. The Handbook of Cognition and Emotion. Sussex, John Wiley \& Sons, Ltd, 45-60.

10. Osgood C, Suci G, Tannenbaum P (1957) The measurement of meaning. Urbana, IL, University of Illinois Press. 
Citation: Torrado M, Silva H, Eusébio S, Fred A, Ouakinin S (2015) Alexithymia, Physiological Reactivity and Cognitive Appraisals of Emotional Stimuli in Opiate Dependents: A Pilot Study.. J Neurol Neurophysiol 6: 263. doi:10.4172/2155-9562.1000263

Page 8 of 8

11. Calder AJ, Burton AM, Miller P, Young AW, Akamatsu S (2001) A principal component analysis of facial expressions. Vision Res 41: 1179-1208.

12. Bradley M, Lang P (2000). Measuring Emotion: behavior, Feeling and Physiology. In Lane R. D., Nadel L. eds. Cognitive Neuroscience of Emotion. Oxford University Press, 242-276).

13. Bradley MM, Lang PJ (2007) The International Affective Picture System (IAPS) in the study of emotion and attention. In Coan J., Allen J. eds. Handbook of Emotion Elicitation and Assessment. Oxford University Press, 29-46.

14. Lang PJ, Greenwald MK, Bradley MM, Hamm AO (1993) Looking at pictures: affective, facial, visceral, and behavioral reactions. Psychophysiology 30: 261-273.

15. Lang PJ, Bradley MM, Cuthbert BN (2008) International affective picture system (IAPS): Affective ratings of pictures and instruction manual. Technical Report A-8. Gainesville, University of Florida.

16. Aguilar de Arcos F, Verdejo-Garcia A, Ceverino A, Montanez-Pareja M Lopez-Juarez E, Sanchez-Barrera M, Lopez-Jimenez A, Perez-Garcia M (2008) Dysregulation of emotional response in current and abstinent heroin users: negative heightening and positive blunting. Psychopharmacology 98:159-166.

17. Gerra G, Baldaro B, Zaimovic A, Moi G, Bussandri M, Raggi MA, Brambilla F (2003) Neuroendocrine responses to experimentally-induced emotions among abstinent opioid-dependent subjects. Drug and Alcohol Dependence 71:25-35

18. Aguilar de Arcos F, Parez M, SÃjnchez MB (2004) Evaluaci An Emocional en Drogodependencias. Sevilla: Junta de AndalucÃa.

19. Wexler BE, Gottschalk CH, Fulbright RK, Prohovnik I, Lacadie CM, et al (2001) Functional magnetic resonance imaging of cocaine craving. Am J Psychiatry 158: 86-95.

20. Schoenbaum G, Roesch MR, Stalnaker TA (2006) Orbitofrontal cortex, decision-making and drug addiction. Trends in Neuroscience 29: 116-124.

21. Larsen JK, Brand N, Bermond B, Hijman R (2003) Cognitive and emotional characteristics of alexithymia: a review of neurobiological studies. J Psychosom Res 54: 533-541.

22. Fukunishi I, Sei H, Morita Y, Rahe RH (1999) Sympathetic activity in alexithymics with mother's low care. J Psychosom Res 46: 579-589.

23. Newton TL, Contrada RJ (1994) Alexithymia and repression: contrasting emotion-focused coping styles. Psychosom Med 56: 457-462.

24. Friedlander L, Lumley MA, Farchione T, Doyal G (1997) Testing the alexithymia hypothesis: physiological and subjective responses during relaxation and stress. J Nerv Ment Dis 185: 233-239.

25. Neumann SA, Sollers JJ, Thayer JF, Waldstein SR (2004) Alexithymia predicts attenuated autonomic reactivity, but prolonged recovery to anger recall in young women. International Journal of Psychophysiology 53: 183-195.

26. Zhao M, Fan C, Du J, Jiang H, Chen H, et al. (2012) Cue-induced craving and physiological reactions in recently and long-abstinent heroindependent patients. Addict Behav 37: 393-398.
27. Chang L, Lin Y, Kuo T, Ho Y, Chen S et al. (2012) Cardiac autonomic modulation during methadone therapy among heroin users: A pilot study. Progress in NeuroPsychopharmacology \& Biological Psychiatry 37: 188-193.

28. Prazeres N, Parker D, Taylor J (2000) Adapta A Portuguesa da Escala de Alexitimia de Toronto de 20 Itens (TAS-20). Revista Iberoamericana de Diagnostico y Evaluati $\tilde{A}^{3}$ n PsicolAgica 9:9-21.

29. Guerreiro MG (1998) Contributo da neuropsicologia para o estudo das

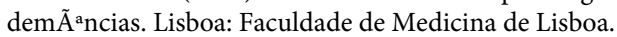

30. Pais-Ribeiro J, Silva I, Ferreira T, Martins A, Meneses R, et al. (2007) Validation study of a Portuguese version of the Hospital Anxiety and Depression Scale. Psychol Health Med 12: 225-235.

31. Lang PJ (1980) Behavioral treatment and bio-behavioral assessment: Computer applications. In Sidowski JB, Johnson JH, Williams TA. eds. Technology in mental health care delivery systems, Norwood, Ablex 119-137.

32. Kreibig SD, Wilhelm FH, Roth WT, Gross JJ (2007) Cardiovascular, electrodermal, and respiratory response patterns to fear- and sadnessinducing films. Psychophysiology 44: 787-806.

33. Kreibig SD (2010) Autonomic nervous system activity in emotion: a review. Biol Psychol 84: 394-421.

34. Oppenheim AV, Schafer RW (2009) Discrete-Time Signal Processing, Prentice Hall.

35. Gustafsson F (1996) Determining the initial states in forward-backward filtering. IEEE Transactions on Signal Processing 44:988-992.

36. Hair JF (2006) Multivariate Data Analysis.Pearson Education, Prentice Hall.

37. Wehmer F, Brejnak C, Lumley M, Stettner L (1995) Alexithymia and physiological reactivity to emotion-provoking visual scenes. J Nerv Ment Dis 183: 351-357.

38. Khantzian EJ (1999) Treating addiction as human process. Northvale: Jason Aronson.

39. Khantzian EJ (2003) Understanding addictive vulnerability. Neuropsychoanalysis 5:5-21.

40. Suh JJ, Ruffins S, Robins CE, Albanese MJ, Khantzian EJ (2008) Selfmedication hypothesis: connecting affective experience and drug choice. Psychoanalytic Psychology 25:518-532.

41. Thayer JF, Ahs F, Fredrikson M, Sollers JJ 3rd, Wager TD (2012) A metaanalysis of heart rate variability and neuroimaging studies: implications for heart rate variability as a marker of stress and health. Neurosci Biobehav Rev 36: 747-756.

42. Verdejo-García A, Bechara A (2009) A somatic marker theory of addiction. Neuropharmacology 56 Suppl 1: 48-62.

43. Savvas SM, Somogyi AA, White JM (2012) The effect of methadone on emotional reactivity. Addiction 107: 388-392.

44. Azizi A, Borjali A, Golzari M (2010) The effectiveness of emotion regulation training and cognitive therapy on the emotional and addictional problems of substance abusers. Iran J Psychiatry 5: 60-65. 